\title{
Effet de l'absorption d'eau sur la réponse mécanique en cisaillement d'un composite (tissus de verre/résine époxyde)
}

\author{
AbDelghani NACERI ${ }^{\mathrm{a}}$ \\ Laboratoire de Matériaux, Faculté des Sciences de l'Ingénieur, Université de M'sila, BP 166, Ichbilia, M'sila 28000, Algérie
}

Reçu le 15 mars 2007, accepté le 7 août 2008

\begin{abstract}
Résumé - Cette étude présente l'analyse de la réponse mécanique en cisaillement d'un stratifié constitué de 12 plis de tissus de fibres de verre $\mathrm{E} /$ résine époxyde conditionné en milieu humide à différents taux d'humidité relative 0,60 et $96 \% \mathrm{RH}$ à $60{ }^{\circ} \mathrm{C}$. L'analyse du comportement mécanique a permis de montrer que l'influence de la concentration en eau sur les caractéristiques mécaniques présente une réduction importante et significative du module de cisaillement et de la contrainte de cisaillement avec la variation de la quantité d'eau absorbée. Cette chute est importante pour les éprouvettes conditionnées à $96 \%$ d'humidité relative. La loi de décroissance exponentielle proposée du module de cisaillement permet de prendre en compte la sensibilité de la réponse mécanique en cisaillement en environnement humide et l'état de déformation de glissement.
\end{abstract}

Mots clés : Absorption d'eau / comportement mécanique / composite / matrice organique

\begin{abstract}
Effect of the moisture absorption on the mechanical response in shear of composite (glass woven fabric/epoxy resin). This study considers the analysis of mechanical response in shear of a laminate constituted of 12 layers of glass fiber fabric/epoxy resin satured at different relative humidies 0,60 and $96 \% \mathrm{RH}$ at $60{ }^{\circ} \mathrm{C}$. The analysis of the mechanical behaviour in shear has permited to show that the influence of the moisture concentration on the mechanical characteristics presents a significant reduction of the shear modulus and the shear stress with the variation of the quantity of water absorption. This reduction is important for the specimens conditioned at $96 \% \mathrm{RH}$. The exponential law proposed for the response in shear has plastic nature and refers to the plastic flow of the matrix. This model precises the evolution of the tangent shear modulus depending on the mechanical state characterized by the shear strain.
\end{abstract}

Key words: Moisture absorption / mechanical behaviour / composite / organic matrix

\section{Introduction}

L'aspect le plus délicat du comportement mécanique des matériaux composites concerne leurs propriétés vis-àvis du cisaillement. En effet, dans ce type de sollicitation, la matrice intervient de manière prépondérante et l'on observe une raideur et une résistance très inférieures à celles observées dans le sens des fibres, ainsi qu'un comportement non-linéaire très marqué.

La présence d'eau au sein des composites fibres/ polymère provoque une détérioration des propriétés mécaniques [1-3]. Ceci souligne la nécessité d'étudier l'effet de l'environnement, caractérisé par les paramètres suivants : température, humidité relative, ou rayonnement

\footnotetext{
a Auteur pour correspondance :

abdelghani_naceri@yahoo.fr
}

ultra-violet, vis-à-vis de la stabilité à moyen et à long terme des structures composites.

On sait qu'au cours d'un vieillissement hygrothermique, la présence de molécules d'eau dans un matériau composite à matrice polymère peut notamment dégrader les liaisons à l'interface renfort-matrice et entraîner ainsi une dégradation irréversible des propriétés du composite $[4,5]$.

Plusieurs études $[6,7]$ ont démontré l'importance des effets que peut entraîner l'absorption d'eau sur les propriétés physiques de composites à matrice organique et sur leur durée de vie. En effet la dégradation d'un matériau pendant son utilisation met en jeu de nombreux paramètres liés à l'environnement et aux sollicitations mécaniques. Ce sont surtout les applications aéronautiques qui ont initié l'étude des problèmes liés à l'environnement $[8,9]$. Il est indispensable d'acquérir une bonne compréhension du comportement de ces matériaux 
quand ils entrent dans la constitution de pièces soumises à des environnements climatiques sévères et complexes (cyclages en température et en humidité).

La concentration en eau d'un composite [10] dépend essentiellement de :

- la nature des fibres et de la résine,

- la disposition des fibres par rapport à la direction de diffusion,

- et l'écart de concentration en eau entre le composite et le milieu environnant.

Les principales conséquences sur les plans physique et mécanique sont :

- la plastification de la résine (modifications des propriétés moléculaires),

- la création des contraintes internes (gonflement contrarié de la matrice),

- la dégradation des interfaces (zones matérielles de transition) entre le renfort et la matrice,

- le développement des fissures dans la résine (lié à l'hétérogénéité de sa structure moléculaire),

- et l'hydrolyse par l'eau des liaisons de la matrice.

L'objet de cette étude est la caractérisation d'un certain nombre de traits spécifiques marquant le comportement mécanique d'un stratifié à matrice polymère renforcé par fibres. Le fil directeur du présent article sera la distinction entre les deux composantes intervenant dans la réponse mécanique d'un matériau composite stratifié soumis à l'action conjuguée de sollicitations d'origine mécanique thermique et hygroscopique :

- une composante matérielle qui consiste à caractériser la monocouche considérée comme matériau homogène et anisotrope. Le phénomène essentiel intervenant à ce niveau est la possibilité d'écoulement plastique en cisaillement le long des fibres. La modélisation simple de ce comportement s'accorde de manière satisfaisante aux résultats expérimentaux;

- Une composante hygroscopique qui consiste à suivre la reprise de poids du composite à renfort tissu en déterminant les deux paramètres principaux de la cinétique d'absorption d'eau du stratifié (le taux maximal de saturation en eau $M_{\mathrm{m}}$ et le coefficient de diffusion $D$ ) dans le cadre du modèle d'absorption de Fick.

\section{Matériau et méthodes expérimentales}

L'étude porte sur un stratifié constitué de 12 plis de tissus à armure taffetas ou toile (le fil de chaîne et de trame s'entrecroisent alternativement) de fibres de verre E noyé dans une résine époxyde holt melt 937/EP/ES67. Ce matériau a été réalisé par la société Hexcel Génin pour le compte du GRECO «Comportement Mécanique des Composites à Fibres ». Il est délivré sous forme de plaques planes de dimensions moyennes : 300 (sens trame) $\times 300$ (sens chaîne) $\times 3,2 \mathrm{~mm}^{3}$.

Le conditionnement en humidité consiste en :

- un séchage préalable en étuve jusqu'à stabilisation pondérale,
- un séjour en enceinte de climatisation à humidité contrôlée à $60 \%$ et $96 \%$ d'humidité relative, à la température de $60{ }^{\circ} \mathrm{C}$, avec surveillance de la reprise en poids jusqu'à une stabilisation.

Le choix des conditions d'environnement à température et aux taux d'humidité constants (0, 60 et $96 \% \mathrm{RH}$ à $60{ }^{\circ} \mathrm{C}$ ) est essentiellement justifié par les deux considérations suivantes :

- au-delà de $60{ }^{\circ} \mathrm{C}$, les échantillons pouvaient être endommagés,

- la température choisie $60{ }^{\circ} \mathrm{C}$ est largement suffisante pour accélérer la reprise en eau et obtenir un palier de saturation en un temps raisonnable.

Le taux maximal de saturation du matériau a été atteint pour une durée de conditionnement moyenne de l'ordre de six mois.

La fraction volumique des fibres a été déterminée selon la méthode de calcination et trouvée égale à $V_{\mathrm{f}}=55 \%$.

La technique de mesure utilisée pour la détermination de la masse volumique du composite tissu est la méthode pictométrique $\left(\rho_{\mathrm{c}}=1,94 \mathrm{~g} . \mathrm{cm}^{3}\right)$.

Il s'agit d'un composite tissu équilibré $(K=0,5)$ et sa composition pondérale est égale à $50 \%$ dans chaque direction de renforcement fibreux (sens chaîne et trame).

La détermination de la cinétique d'absorption d'eau du matériau étudié a été effectuée par une mesure de reprise de poids en fonction du temps dans le but de déterminer les deux paramètres caractéristiques de la loi de diffusion de Fick qui admet la réversibilité du phénomène :

- le coefficient de diffusion $D$ en $\mathrm{cm}^{2} \cdot \mathrm{s}^{-1}$,

- et la quantité maximale d'eau retenue à la saturation $M_{\mathrm{m}}$ en $\%$.

La cinétique d'absorption d'eau a été suivie par la méthode gravimétrique (reprise de poids mesurée à l'aide d'une balance sartorius 2842 avec une étendue de pesée de $160 \mathrm{~g}$ et une précision de 0,1 mg) afin de contrôler le gain en poids du matériau conditionné en fonction de la durée de conditionnement.

L'essai mécanique consiste à soumettre les échantillons à une rampe monotone en traction uniaxiale et analyser l'effet du conditionnement en humidité sur la réponse mécanique.

Le choix de l'essai de traction uniaxiale monotone comme essai de caractérisation mécanique est justifié par le fait qu'il reste d'interprétation relativement aisée pour les matériaux composites.

L'essai est mené sur des éprouvettes usinées avec un rayon de $1000 \mathrm{~mm}$ de longueur totale $200 \mathrm{~mm}$, d'épaisseur $3,2 \mathrm{~mm}$, de largeur à l'extrémité de $30 \mathrm{~mm}$ et au centre de $20 \mathrm{~mm}$ (Fig. 1). Afin d'éviter l'endommagement des éprouvettes dans les mors, une forme géométrique profilée « poutre de type haltère » avec un rayon de $1000 \mathrm{~mm}$ a été adoptée. Le choix de ce type d'éprouvette qui présente une section réduite au centre est justifié compte tenu du fait que le développement de l'endommagement reste 


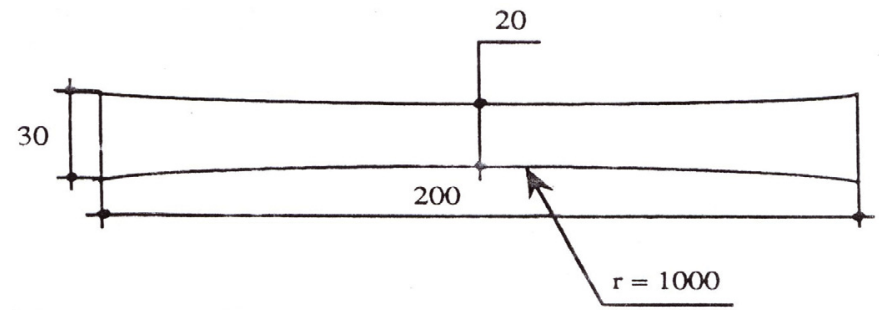

Fig. 1. Éprouvette usinée avec un rayon de $1000 \mathrm{~mm}$.

bien localisé dans la zone centrale sans qu'il en résulte pour autant de réduction significative des caractéristiques mécaniques ultimes. Le détail de ce choix est discuté dans la référence [11].

Les échantillons équipés de jauges de déformation et de talons ont été soumis à un essai de traction unaxiale en rampe monotone sur une machine de traction servohydraulique de type MST 810 de capacité \pm 100 KN (équipée de mors auto-serrants), contrôlé en vitesse de déplacement imposé constante $\left(V=0,66 \mathrm{~mm} \cdot \mathrm{min}^{-1}\right)$.

\section{Résultats expérimentaux}

\subsection{Cinétique d'absorption d'eau}

Le calcul du coefficient de diffusion $D$ et l'estimation de la quantité maximale d'eau retenue à la saturation $M_{\mathrm{m}}$ du composite sont effectués à partir de la courbe maîtresse présentant l'évolution de la quantité d'eau absorbée $M$ en fonction de la racine carrée du temps $(\sqrt{t})$.

Le taux maximal de saturation du matériau a été atteint pour une durée de conditionnement moyenne de l'ordre de six mois.

Le gain en poids $M$ dépend principalement de trois paramètres $t, M_{\mathrm{m}}$ et $D$.

$$
M(\%)=\frac{W-W 0}{W 0} 100
$$

où :

$M$ : gain en poids (quantité d'eau absorbée),

$W$ : poids à l'instant $t$,

$W 0$ : poids à l'état sec (initial).

Le calcul de la diffusivité $D$ peut être effectué très simplement à partir de la pente de la partie linéaire de la courbe de reprise de poids et de la valeur asymptotique $M_{\mathrm{m}}$ et l'on a :

$$
D=\pi\left[(h / 4) \cdot M_{\mathrm{m}}\right]^{2} \cdot\left[\left(M_{\mathrm{b}}-M_{\mathrm{a}}\right) /\left(\sqrt{t_{\mathrm{b}}}-\sqrt{t_{\mathrm{a}}}\right)\right]^{2}
$$

avec :

$D$ : coefficient de diffusion,

$M_{\mathrm{m}}$ : gain en poids maximum ou quantité maximale d'eau retenue à la saturation,

$h$ : épaisseur du matériau.

Le tableau 1 présente les valeurs des paramètres caractéristiques de la cinétique d'absorption d'eau (la quantité maximale d'eau retenue à la saturation $M_{\mathrm{m}}$ et le
Tableau 1. Résultats expérimentaux d'absorption d'eau $\left(M_{\mathrm{m}}\right.$ et $D$ ) du composite époxyde/verre E à différents taux d'humidité relative à $60^{\circ} \mathrm{C}$.

\begin{tabular}{ccc}
\hline $\begin{array}{c}\text { Conditionnement } \\
\mathrm{RH} \text { en }(\%)\end{array}$ & 60 & 96 \\
\hline $\mathrm{D}$ \\
$\mathrm{en} \mathrm{cm}^{2} \cdot \mathrm{s}^{-1} \cdot 10^{-8}$ & 1,200 & 0,210 \\
\hline $\begin{array}{c}M_{\mathrm{m}} \\
\text { en }(\%) \mathrm{du} \text { composite }\end{array}$ & 0,168 & 1,000 \\
\hline
\end{tabular}

Tableau 2. Paramètres caractéristiques de la cinétique d'absorption d'eau du composite polyester/verre E à différents taux d'humidité relative à $65^{\circ} \mathrm{C}[12]$.

\begin{tabular}{ccc}
\hline $\begin{array}{c}\text { Humidité relative }(\mathrm{RH}) \text { en } \\
\%\end{array}$ & $\begin{array}{c}M_{\mathrm{m}} \\
\text { en } \%\end{array}$ & $\begin{array}{c}D \\
\text { en } \mathrm{cm}^{2} \cdot \mathrm{s}^{-1} \cdot 10^{-8}\end{array}$ \\
\hline 60 & 0,45 & 23,0 \\
100 & 2,75 & 3,80 \\
\hline
\end{tabular}

coefficient de diffusion $D$ ) obtenues par l'essai de conditionnement hygrothermique réalisé au laboratoire, celui-ci confirme clairement les remarques principales observées :

- le coefficient de diffusion $D$ dépend seulement de la température mais indépendamment de l'humidité relative,

- et la quantité maximale d'eau retenue $M_{\mathrm{m}}$ à la saturation dépend fortement du taux d'humidité relative.

Les résultats de la cinétique d'absorption d'eau (Tab. 2) obtenues par Loos et Springer [12] du composite polyester/verre E (SMC-30EA) vont dans le même sens que nos valeurs expérimentales d'absorption d'eau $\left(M_{\mathrm{m}}\right.$ et $\left.D\right)$. Cette comparaison nous permet de dire que les deux paramètres principaux de diffusion $\left(M_{\mathrm{m}}\right.$ et $\left.D\right)$ dépendent non seulement de la température et du taux d'humidité relative mais aussi très fortement de la nature du matériau (type de résine, fibres, etc.).

Le coefficient de diffusion $D$ à $60 \%$ RH est nettement supérieur par rapport à celui obtenu pour le conditionnement hygrothermique réalisé à $96 \% \mathrm{RH}$, ceci est due essentiellement à la variation de la vitesse de la cinétique d'absorption d'eau (plus l'humidité relative est basse plus la cinétique d'absorption d'eau est rapide, ceci est dû à la dilatation du matériau qui augmente le volume disponible pour l'accès de l'eau). L'eau est absorbée par les couches externes, puis diffuse dans tout le matériau.

La fixation des molécules d'eau sur les chaînes moléculaires a deux conséquences majeures : la diminution de la température de transition vitreuse et le gonflement du polymère.

La présence d'eau dans le réseau macromoléculaire a de nombreuses conséquences sur les propriétés physicochimiques et mécaniques du polymère et, par voie de conséquence, du composite. Les modifications peuvent être réversibles, plastification de la matrice polymère, ou irréversibles, hydrolyse, dégradation des interfaces (zones matérielles de transition) entre le renfort et la matrice et microfissuration. 
Tableau 3. Résultats mécaniques en cisaillement.

\begin{tabular}{cccc}
\hline $\begin{array}{c}\text { Humidités } \\
\text { Relatives }\end{array}$ & $\begin{array}{c}0 \% \mathrm{RH} \\
\text { à } 60{ }^{\circ} \mathrm{C}\end{array}$ & $\begin{array}{c}60 \% \mathrm{RH} \\
\text { à } 60{ }^{\circ} \mathrm{C}\end{array}$ & $\begin{array}{c}96 \% \mathrm{RH} \\
\text { à } 60{ }^{\circ} \mathrm{C}\end{array}$ \\
\hline $\begin{array}{c}G_{06} \\
(\mathrm{GPa})\end{array}$ & 6,08 & 5,55 & 3,49 \\
\hline $\begin{array}{c}\sigma_{6 \text { ultime }} \\
(\mathrm{MPa})\end{array}$ & 145 & 128 & 100 \\
\hline $\begin{array}{c}\varepsilon_{6 \text { ultime }} \\
(\%)\end{array}$ & 15,83 & 10,65 & 9,70 \\
\hline
\end{tabular}

\subsection{Essais mécaniques}

La reprise d'eau (phénomène de vieillissement) entraîne une diminution importante et significative du module de cisaillement tangent, du glissement et de la contrainte de cisaillement (Tab. 3) avec la variation de la quantité d'eau absorbée (influence très marquante de l'humidité) sur le matériau testé.

L'influence de l'absorption d'eau se traduit par une chute des caractéristiques mécaniques, cette réduction est considérable pour les éprouvettes conditionnées à $96 \%$ $\mathrm{RH}$.

L'apparition du phénomène de non-linéarité de nature plastique commence dès le début de l'essai de traction uniaxiale «essai à $\pm 45^{\circ} »$ pour des valeurs très faibles de déformation de cisaillement, sans domaine élastique significatif.

L'évolution du module de cisaillement tangent présente une légère croissance à la fin de l'essai pour un niveau de glissement supérieur à $4,5 \%$ qui représente un comportement différent par rapport à celui d'un stratifié $\pm 45^{\circ}$ [13], ce phénomène peut être dû aux frottements de fibres tissées entre la chaîne et la trame et à la dégradation des interfaces (zones matérielles de transition) entre le renfort et la matrice.

La remarque principale que l'on peut faire est l'influence déterminante de la teneur en eau du matériau sur la valeur initiale de $G_{06}$ et sur la décroissance qui est gouvernée par $\gamma_{06}$.

\section{Modélisation}

La non-linéarité de la réponse mécanique de cisaillement observée lors de l'essai de traction uniaxiale des éprouvettes testées « essai à $\pm 45^{\circ} »$ dans différentes conditions d'environnement est de nature «plastique » car elle est liée principalement à l'état cinématique du matériau caractérisé par la déformation de cisaillement.

Le modèle simple proposé pour la caractérisation du comportement mécanique en cisaillement du stratifié tissu testé est représenté par une loi exponentielle de décroissance du module de cisaillement tangent en fonction du glissement :

$$
\frac{G 6-G 1}{G 06-G 1}=\exp \left(-\frac{\gamma 6}{\gamma 06}\right)
$$

Tableau 4. Résultats des paramètres principaux du modèle proposé.

\begin{tabular}{ccccc}
\hline $\begin{array}{c}\text { RH en }(\%) \\
\text { à } 60{ }^{\circ} \mathrm{C}\end{array}$ & $M_{\mathrm{m}}$ & $G_{1}$ & $G_{06}$ & $\gamma_{06}$ \\
\hline $0 \% \mathrm{RH}$ & 0,000 & 0,080 & 6,080 & 0,620 \\
\hline $60 \% \mathrm{RH}$ & 0,168 & 0,180 & 5,550 & 0,550 \\
\hline $96 \% \mathrm{RH}$ & 1,000 & 0,200 & 3,490 & 0,430 \\
\hline
\end{tabular}

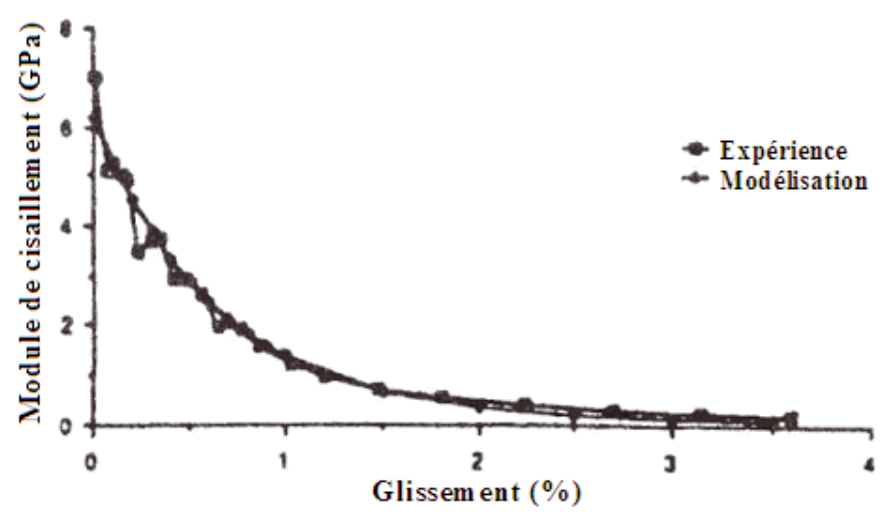

Fig. 2. Réponses expérimentale et modélisée.

où :

$G_{06}$ : module initial tangent de cisaillement en GPa,

$G_{1}$ : valeur asymptotique de la fonction exponentielle décroissante en GPa,

$G_{6}$ : module de cisaillement en GPa,

$\gamma_{06}:$ glissement caractéristique en $\%$,

$\gamma_{6}$ : déformation due au cisaillement en $\%$.

L'identification des paramètres matériels de la réponse mécanique en cisaillement qui confirme le bien-fondé du modèle proposé consiste à déterminer les valeurs de $G_{06}$, $G_{1}$ et de $\gamma_{06}$ (Tab. 4).

Le tracé de la fonction de décroissance du module de cisaillement en échelle logarithmique permet de déterminer les valeurs des paramètres caractérisant le modèle proposé.

* $-\frac{1}{\gamma_{06}}$ : représente la pente de la droite de la fonction en échelle logarithmique,

* $\log \left(G_{06}-G_{1}\right)$ : représente la valeur initiale (intersection de la droite avec l'axe des ordonnées pour $\gamma_{6}=$ $0)$ du module tangent de cisaillement de la fonction en échelle logarithmique.

La détermination de la valeur asymptotique $G_{1}$ quand $\gamma_{6}$ tend vers l'infini de la fonction exponentielle décroissante est très difficile à déterminer avec précision comme toute valeur asymptotique.

La bonne concordance entre les courbes tracées (modélisée et expérimentale) du modèle proposé du comportement en cisaillement justifie le bon contrôle de la non-linéarité de la réponse mécanique en cisaillement du matériau testé (Fig. 2). 


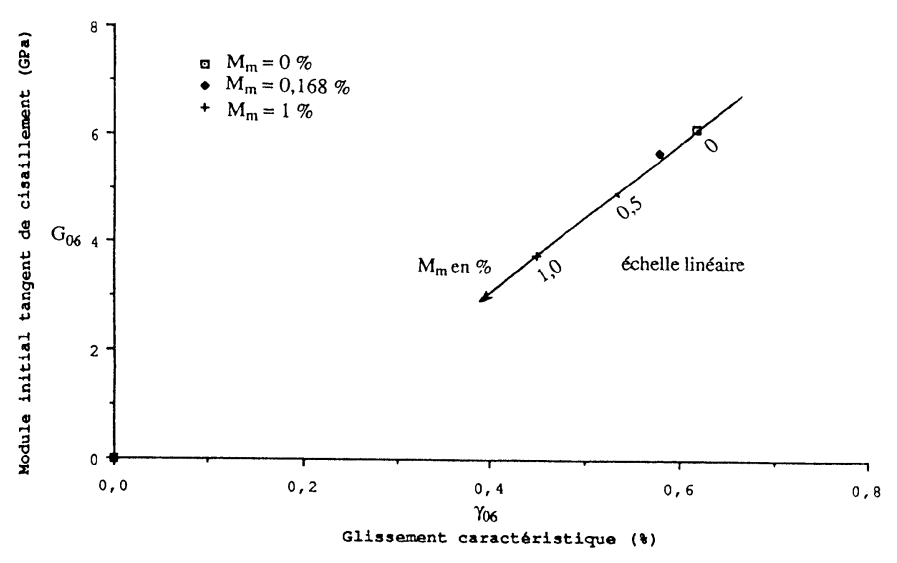

Fig. 3. Module initial tangent de cisaillement $\left(G_{06}\right)$ en fonction du glissement caractéristique $\left(\gamma_{06}\right)$.

Le tracé de la courbe (Fig. 3) représentant le module initial tangent de cisaillement en fonction du glissement caractéristique en tenant compte de la concentration maximale d'eau retenue pour les échantillons conditionnés à différents taux d'humidité relative $(0,60$ et $96 \% \mathrm{RH}$ à $60{ }^{\circ} \mathrm{C}$ ), nous a permis de proposer un modèle simple de la droite obtenue permettant de prendre en considération la sensibilité du comportement en cisaillement à l'humidité et à l'état de déformation.

L'expression proposée de la droite obtenue est la suivante :

$$
G_{06}=a \gamma_{06}-b
$$

où :

$a$ : représente la pente de la droite de la fonction en échelle linéaire,

$-b$ : représente la valeur initiale (intersection de la droite avec l'axe des ordonnées pour $\gamma_{06}=0$ ) du module de cisaillement de la fonction en échelle linéaire.

La graduation de la droite (Fig. 3) en échelle linéaire de $M_{\mathrm{m}}$ qui correspond à une échelle bien définie, permet de préciser pour chaque quantité d'eau absorbée la valeur du module initial tangent de cisaillement et du glissement caractéristique.

La courbe tracée (Fig. 4) représentant le glissement caractéristique en fonction de la quantité maximale d'eau retenue à la saturation par le matériau permet de déterminer les coefficients caractéristiques $a^{\prime}$ et $b^{\prime}$ d'après l'équation de la droite obtenue :

$$
\gamma_{06}=b^{\prime}-a^{\prime} M_{\mathrm{m}}
$$

avec :

$M_{\mathrm{m}}$ : quantité maximale d'eau retenue à la saturation en $\%$,

$-a^{\prime}$ : représente la pente de la droite de la fonction en échelle linéaire,

$b^{\prime}$ : représente la valeur initiale (intersection de la droite avec l'axe des ordonnées pour $M_{\mathrm{m}}=0$ ) du glissement caractéristique de la fonction en échelle linéaire.

Cette modélisation permet donc de tenir en compte de l'influence de l'absorption d'eau sur le comportement mécanique en cisaillement.

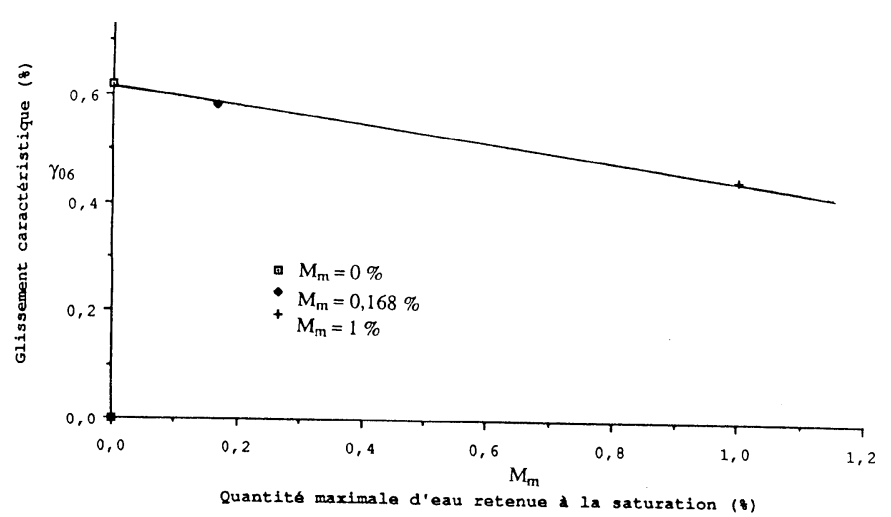

Fig. 4. Glissement caractéristique $\left(\gamma_{06}\right)$ en fonction de la concentration maximale d'eau retenue $\left(M_{\mathrm{m}}\right)$.

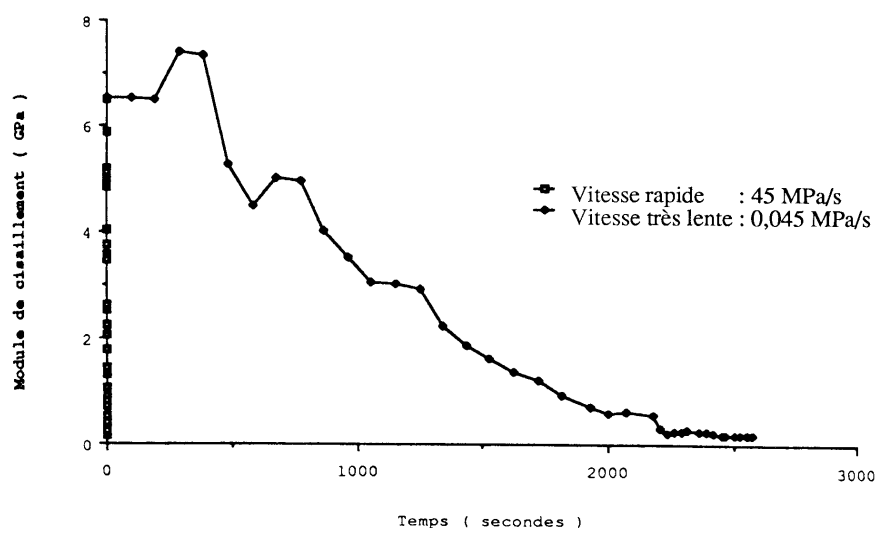

Fig. 5. Module tangent de cisaillement en fonction du temps.

La confirmation du modèle plastique proposé a été précisée par les courbes (Figs. 5 et 6) du module tangent de cisaillement en fonction du glissement et du temps pour deux vitesses différentes de chargement (rapide et très lente) en rejetant l'hypothèse de la viscoélasticité qui dépend principalement du temps (les courbes expérimentales devraient être superposables dans la représentation en fonction du temps). Pour un modèle viscoélastique linéaire le module de cisaillement est celui de relaxation. Il est clair aussi que l'écart observé (Fig. 5) est très important pour qu'un recours à une théorie viscoélastique non-linéaire soit suffisant.

On constate aussi d'après le tracé présenté (Fig. 6) que la variable dont dépend le module tangent de cisaillement est non pas le temps mais le glissement c'est-à-dire que la viscoélasticité n'est pas responsable de la non-linéarité du comportement en cisaillement. La représentation du module tangent de cisaillement en fonction du glissement permet de préciser clairement que le comportement mécanique en cisaillement ne dépend pas de la vitesse d'essai (les deux courbes sont confondues).

L'apparition du phénomène de non-linéarité de nature plastique (Fig. 6) commence dès le début de l'essai de traction uniaxiale « essai à $\pm 45^{\circ}$ » pour des valeurs 


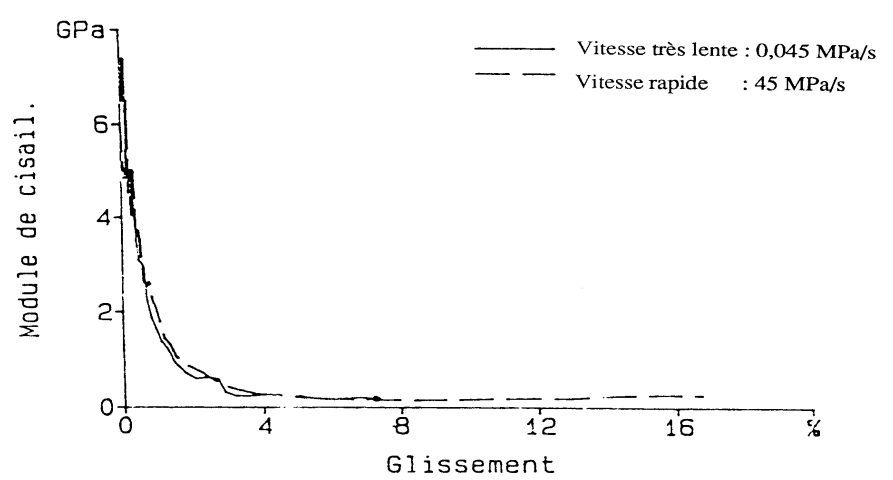

Fig. 6. Module tangent de cisaillement en fonction du glissement.

très faibles de déformation de cisaillement, sans domaine élastique significatif.

\section{Conclusion}

L'analyse des résultats expérimentaux obtenus du composite tissu de verre $\mathrm{E} /$ résine époxyde conditionné en milieu vapeur a permis de tirer les conclusions suivantes :

* Réduction importante et significative de l'ordre de $9 \%, 33 \%$ et $12 \%$ à $60 \% \mathrm{RH}$ et de $43 \%, 39 \%$ et $31 \%$ à $96 \% \mathrm{RH}$ respectivement pour le module de cisaillement tangent, le glissement et la contrainte de cisaillement avec la variation de la quantité d'eau absorbée.

* L'évolution du module de cisaillement tangent présente une légère croissance à la fin de l'essai pour un niveau de glissement supérieur à 4,5\% qui représente un comportement différent par rapport à celui d'un stratifié $\pm 45^{\circ}$ [13], ce phénomène peut être dû aux frottements de fibres tissées entre la chaîne et la trame et à la dégradation des interfaces (zones matérielles de transition) entre le renfort et la matrice.

* La loi de décroissance exponentielle proposée du module de cisaillement tangent permet de prendre en compte l'influence de la concentration en eau sur la réponse mécanique en cisaillement et de l'état de déformation de glissement du matériau testé.

* La réponse non-linéaire du comportement mécanique en cisaillement pourrait être due à l'écoulement plastique de la matrice qui dépend principalement de l'état cinématique du matériau (déformation plastique de cisaillement).

Les résultats présentés permettent de conclure quant à la question posée : la réponse de ce composite ne peut pas s'expliquer dans le cadre de la visco-élasticité dans le domaine de déformation considéré. Le mot « linéaire »a été omis dans cette conclusion car nous pensons que les différences observées sur l'aspect des courbes représentées en fonction du temps ne peuvent pas être considérées comme étant du premier ordre (comme il le faudrait dans le cas de la viscoélasticité non-linéaire), mais que bien plus fondamentalement elles découlent du fait que le temps n'est pas la bonne variable à faire intervenir. Le fait qu'une description en fonction d'une variable cinématique (donc liée à l'état du matériau) semble mieux adaptée amène à la conclusion que la plasticité est le phénomène prépondérant qui gouverne la réponse en cisaillement des composites à matrice polymère.

Une analyse plus complète de l'absorption de l'eau et de ces conséquences reste à faire sur des matériaux composites utilisant le même type de résine, mais des renforcements différents (renforts unidirectionnels, stratifiés, ...) afin de préciser le rôle de chaque constituant.

\section{Références}

[1] G.S. Springer, Environmental Effects on Composites Materials, ed. G.S. Springer, Technomic, Westport, 1 (1981) 1-6

[2] Y.C. Zchang, X. Wang, Hygrothermal effects on interfacial stress transfer characteristics of carbon nanotubesreinforced composites system, J. Reinforced Plastics and Composites 25 (2006) 71-88

[3] A. Bergeret, I. Pires, M. Arpin, J.P. Gasca, A. Crespy, Stability of glass fiber reinforced polyamide 66 in a service-related environment: Influence of the fiber/matrix interface, Eurofillers, Lodz, Pologne, 2001

[4] I. Verpoest, G.S. Springer, Moisture absorption characteristics of aramid-epoxy composites, J. Reinforced Plastics and Composites 17 (1988) 2-32

[5] A. Chateauminois, B. Chabert, J.P. Soulier, L. Vincent, Hygrothermal ageing effects on the static fatigue of glass/epoxy composites, Composites 7 (1998) 547-555

[6] M.P. Foulc, A. Bergeret, L. Ferry, A. Crespy, Étude $\mathrm{du}$ vieillissement hygrothermique de matériau composites polyéthylène téréphtalate (PET) - fibres de verre, Journées d'Études des Polymères, Metz, France, 2000

[7] G. Camino, M.P. Luda, A.Y. Polishchuk, M. Revellino, R. Blancon, G. Merle, J.J. Martinez-Vega, Kinetic aspects of water absorption in polyster resin glass fibers composites, Composites Science and Technology 57 (1997) 1469-1482

[8] M.P. Foulc, A. Bergeret, L. Ferry, L. Panaiva, A. Perichaud, A. Crespy, Comprehension des mécanismes de vieillissement hygrothermique de composites PET - fibres de verre, Journée du G.F.P Méditerranée, Marseille, 2001

[9] L. Mc Kague, Environmental synergism and simulation in resin matrix composites, ASTM-STP 658 (1978) 193-204

[10] D. Colombini, J.J. Martinez-Vega, G. Merle, Influence of hygrothermal ageing and thermal treaments on the viscoelastic behaviour of DGEBA-MCDEA epoxy resin, Polymer 43 (2001) 1983-1992

[11] A. Naceri, A. Vautrin, Proposition d'un modèle expérimental pour la caractérisation de la réponse mécanique d'un composite (tissu de verre/résine époxyde), J. Phys. IV 124 (2005) 201-206

[12] A.C. Loos, G.S. Springer, Moisture absorption of polyester glass composites, Environmental Effects on, Composite Materials, Technomic, Westport 1 (1981) $51-62$

[13] Y. Surrel, A. Vautrin, On a modeling of the plastic response of FRP under monotonic loading, J. Composite Mat. 23 (1989) 232-250 